\title{
Optical Injection-Induced Timing Jitter Reduction in Gain-Switched Single-Mode Vertical-Cavity Surface-Emitting Lasers
}

\author{
A. Consoli ${ }^{\mathrm{a}}$, J. M. Noriega ${ }^{\mathrm{b}}$, A. Valle ${ }^{\mathrm{c}}$ L. Pesquera*c ${ }^{*}$ I. Esquivias ${ }^{\mathrm{a}}$, and F. J. López -Hernández ${ }^{\mathrm{a}}$ \\ ${ }^{a}$ Departamento de Tecnología Fotónica, Universidad Politécnica de Madrid, Madrid 28040, Spain; \\ ${ }^{\mathrm{b}}$ Departamento de Matemáticas, Facultad de Ciencias, Universidad de Oviedo, C/ Calvo Sotelo s/n, \\ 33007, Oviedo, Spain; \\ 'Instituto de Física de Cantabria, CSIC-Universidad de Cantabria, Edificio Juan Jordá, Avda. Los \\ Castros s/n, E-39005, Santander, Spain
}

\begin{abstract}
We report an experimental and theoretical investigation of the effect of optical injection on the characteristics of optical pulses generated by gain-switching a $1550 \mathrm{~nm}$ single transverse mode vertical-cavity surface-emitting laser (VCSEL). Under continuous wave operation the VCSEL emits in a linear polarization along the whole current range. The experimental analysis of the effect of external optical injection on the timing jitter, maximum power, and pulse width of optical pulses generated by gain-switching the single mode VCSEL is performed for several repetition rates and for different values of the detuning between the frequency of the optical injection and the VCSEL. Experimental results show that for $1 \mathrm{GHz}$ repetition frequency, jitter reductions greater than $70 \%$ can be obtained over a $47 \mathrm{GHz}$ frequency detuning range with a slight increase of $22 \%$ in pulse width with respect to the solitary case. A clear anticorrelation between the maximum power and pulse width is also obtained. A theoretical study is also performed by using a model that incorporates both spatial dependence of carrier density and optical field profiles. The two polarization modes are also taken into account in the model. The theoretical results are in good agreement with the experimental results.
\end{abstract}

Keywords: Optical injection, gain-switching, timing jitter, vertical-cavity surface-emitting laser (VCSEL)

\section{INTRODUCTION}

Injection locking is a technique commonly employed to improve the semiconductor laser performance. Laser spectral narrowing, suppression of laser noise, reduction of frequency chirp under modulation and improvement of the laser intrinsic frequency response have been obtained by using that technique. Such studies were extended to vertical-cavity surface-emitting lasers (VCSELs) [1]-[2] which present significant advantages over their edge-emitting counterparts, including a low threshold current, single-longitudinal-mode operation and wafer-scale integrability [3]. VCSELs are attractive for use in injection locking because of their compactness, low power consumption, and circular output beam [3]. Injection in VCSELs has been also used for performing several all-optical signal processing functions like all-optical inversion and all-optical regeneration [3]. $1550 \mathrm{~nm}$ VCSELs have been recognized as potential low-cost high-quality signal light sources compared to the conventional distributed feedback lasers (DFB) for optical communication systems [4]. A low-cost $1550 \mathrm{~nm}$ semiconductor laser is important for fiber-to-the-home applications, especially with the wavelength-division-multiplexing (WDM) or optical code division multiplexing (OCDMA) access systems utilizing $1550 \mathrm{~nm}$ wavelength regions [5,6]. Short optical pulse generation at $1550 \mathrm{~nm}$ from a low-cost commercial gain-switched VCSEL was recently reported [7]. As the pulse width becomes shorter and shorter timing jitter becomes a serious factor in determining the performance of the VCSEL. Short optical pulses with small timing jitter are desirable as data signals in high-frequency optical communication systems. Timing jitter reduction has been achieved by using optical injection in gain-switched DFB lasers [8]-[9]. A similar study has been recently considered in VCSELs in a theoretical way [10]. Numerical simulations indicate that jitter reductions greater than $50 \%$ can be obtained with a slight increase in pulse width (smaller than $25 \%$ ) [10]. 
In this paper we investigate experimentally the effect of external optical injection on the timing jitter, maximum power, and pulse width of optical pulses generated by gain-switching a single mode VCSEL at several repetition rates. A theoretical study is also performed by extending previous analysis [10] to the repetition rate of $1 \mathrm{GHz}$ considered in the experiments. The two polarizations of the fundamental transverse mode of the VCSEL are also taken into account in the model. The paper is organized as follows. In Section 2 we describe the experimental set-up and the solitary VCSEL characteristics. Experimental results are described in Section 3. Section 4 is devoted to the theoretical results. Finally, Section 4 contains the conclusions.

\section{EXPERIMENTAL SET-UP}

Optical injection in a gain-switched VCSEL is experimentally achieved by using the setup shown in Fig. 1. An all fiber system has been developed in order to inject the light from a tunable external-cavity laser diode (TL, Tunics Plus-CL) into a quantum-well commercial VCSEL (Raycan) that emits around $1555 \mathrm{~nm}$. The output of the tunable laser is injected into the VCSEL via a three-port polarization maintaining optical circulator (PMOC). The control of the polarization injected into the VCSEL is achieved by using a polarization controller (PC). Gain-switching of the VCSEL is obtained by using a bias- $\mathrm{T}$ that is driven by the bias current generator ( $\mathrm{I}_{\mathrm{BI} A S}$, Keithley 236) and by the pulse generator (PG, Anritsu MP1800A). The light is analyzed by using a $20 \mathrm{GHz}$ communication signal analyzer (CSA, Tektronics CSA8000) and an optical spectrum analyzer with a resolution of $0.07 \mathrm{~nm}$ (OSA, Anritsu MS9710B).

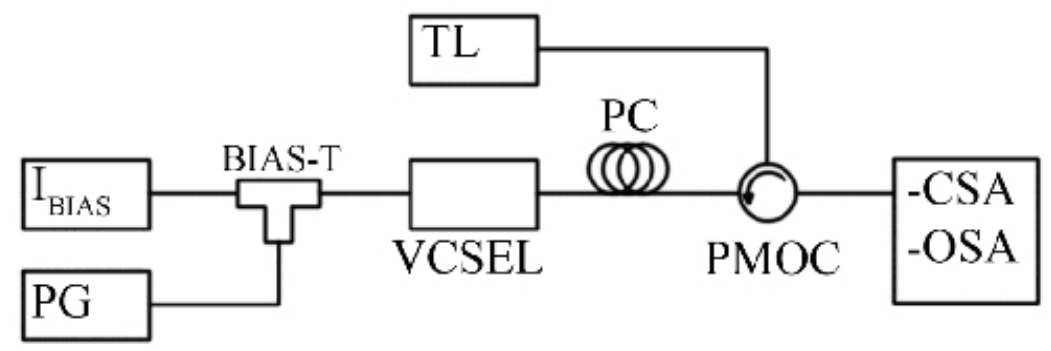

Figure 1. Experimental set-up. TL: tunable external-cavity laser diode; $\mathrm{I}_{\mathrm{BIAS}}$ : bias current generator; PG: Pattern Generator; PC: Polarization Controller; PMOC: Polarization Maintaining Optical Circulator; OSA: Optical Spectrum Analyzer; CSA: communication signal analyzer.

The VCSEL threshold current, $\mathrm{I}_{\text {th }}$, is $2.3 \mathrm{~mA}$. Under continuous wave operation the VCSEL emits in a linearly polarized fundamental transverse mode along the whole current range. The subsidiary orthogonal polarization is suppressed more than $30 \mathrm{~dB}$ and it is shifted $0.48 \mathrm{~nm}$ to the long wavelength side with respect to the dominant one. The solid line of Fig. 2 corresponds to the optical spectrum when the applied bias current is $3 \mathrm{~mA}$. The two peaks that appear at longer wavelengths correspond to the two orthogonal linear polarizations of the fundamental transverse mode. The peak that appears at longer wavelengths corresponds to the depressed polarization (referred as orthogonal polarization) of the fundamental transverse mode. The dominant linear polarization (referred as parallel polarization) appears at a shorter wavelength. Peaks appearing at shorter wavelengths correspond to non lasing higher-order transverse modes. When using the setup of Fig. 1, the PC is adjusted in such a way that the light is injected parallel to the dominant linear polarization. In this situation, the subsidiary orthogonal polarization suffers a maximum attenuation because it orthogonally couples to the preferential direction of the polarization maintaining OC. This is illustrated in Fig. 2, where the dashed line corresponds to the spectrum with the orthogonal polarization suppressed. 


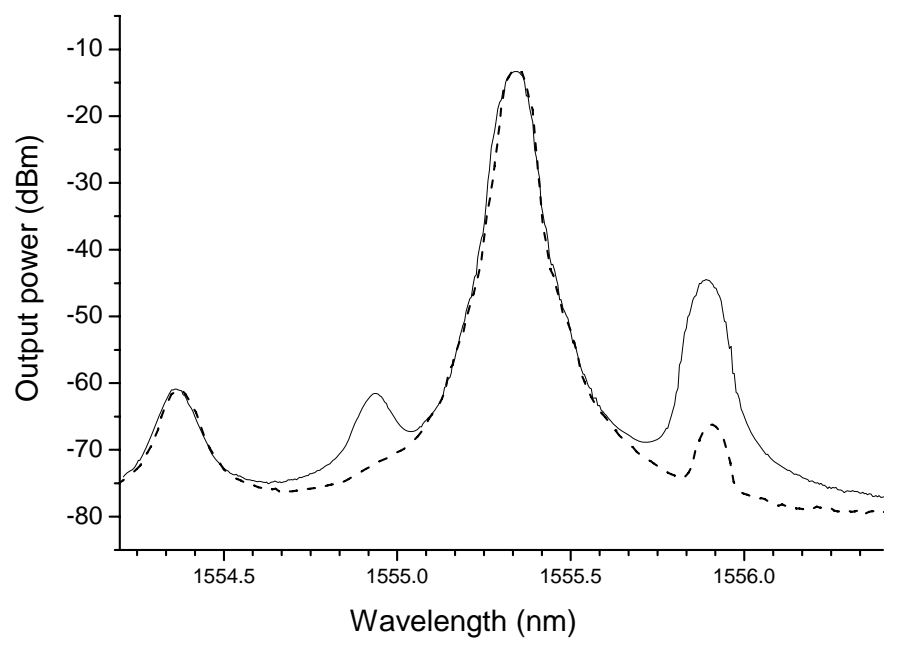

Figure 2. Optical spectrum of the VCSEL for a bias current of $3 \mathrm{~mA}$ without polarization selection (solid line) and with the orthogonal polarization suppressed (dashed line).

Under continuous wave operation the orthogonal polarization is suppressed more than $30 \mathrm{~dB}$ with respect to the parallel polarization of the fundamental transverse mode (see the two peaks at longer wavelengths of the solid line of Fig. 2). However, when the VCSEL is modulated the subsidiary orthogonal polarization is enhanced. We show in Fig. 3 the spectrum obtained when the VCSEL is gain-switched with a repetition rate of $100 \mathrm{MHz}$ under the following conditions: $\mathrm{I}_{\mathrm{BIAS}}=3 \mathrm{~mA}$, modulating amplitude of $2.5 \mathrm{~V}$, pulse width $250 \mathrm{ps}$ and duty cycle of $2.5 \%$. The peak that appears at longer wavelengths corresponds to the orthogonal polarization, that is suppressed only $7 \mathrm{~dB}$ with respect to the parallel polarization (solid line of Fig. 3). Then the side mode suppression ratio is reduced more than $20 \mathrm{~dB}$ due to the gainswitching operation of the VCSEL. The dashed line of Fig. 3 corresponds to the spectrum when the PC is adjusted in such a way that the orthogonal polarization is suppressed.

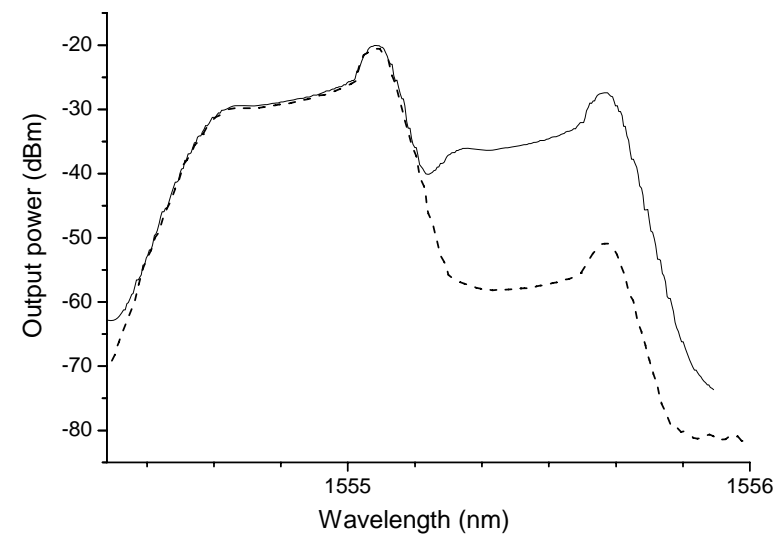

Figure 3. Optical spectrum of the gain-switched VCSEL for a repetition rate of $100 \mathrm{MHz}$ without polarization selection (solid line) and with the orthogonal polarization suppressed (dashed line).

\section{EXPERIMENTAL RESULTS}

We have measured the characteristics of optical pulses generated by gain-switching the VCSEL at several repetition rates. We first consider the case of $1 \mathrm{GHz}$ repetition frequency. A square-wave modulation is applied to the VCSEL with a repetition rate of $1 \mathrm{GHz}$ and duty cycle of $50 \%$ and $\mathrm{I}_{\text {BIAS }}=1.3 \mathrm{I}_{\mathrm{th}}=3 \mathrm{~mA}$. The voltage in the PG is $2.5 \mathrm{~V}$ during 500 
ps and $0 \mathrm{~V}$ during the rest of the pulse. Bias current is set greater than $\mathrm{I}_{\mathrm{th}}$ because the bias-tee suppress the mean value of the pulse signal, and therefore the laser is switched from below threshold to above threshold condition. The optical spectrum of the free-running VCSEL is shown in Fig. 4 (solid line). Note that the PC is adjusted in such a way that the orthogonal polarization is suppressed. Spectral broadening due to chirping effects is obtained [11]. The main peak is the one corresponding to the parallel polarization. The secondary peak appearing at longer wavelengths corresponds to the orthogonal polarization. The optical spectrum of the VCSEL subject to optical injection is also shown in Fig. 4 (dashed line). The optical injection is such that the power of the tunable laser, $\mathrm{P}_{\mathrm{ML}}$, is $1 \mathrm{~mW}$ and its wavelength, $\lambda_{\mathrm{ML}}$, is 1558.26 $\mathrm{nm}$, near to the wavelength of the parallel polarization, $\lambda_{\mid}=1558.3 \mathrm{~nm}$. The corresponding frequency detuning, $\Delta v=v_{\mathrm{ML}}-$ $v_{\|}$, of the injected signal with respect to the parallel polarization is $5 \mathrm{GHz}$. Fig. 4 shows that, as previously reported in DFB lasers [9], optical injection produces a narrowing of the optical spectrum and an improvement of the side mode suppression ratio of $23 \mathrm{~dB}$.

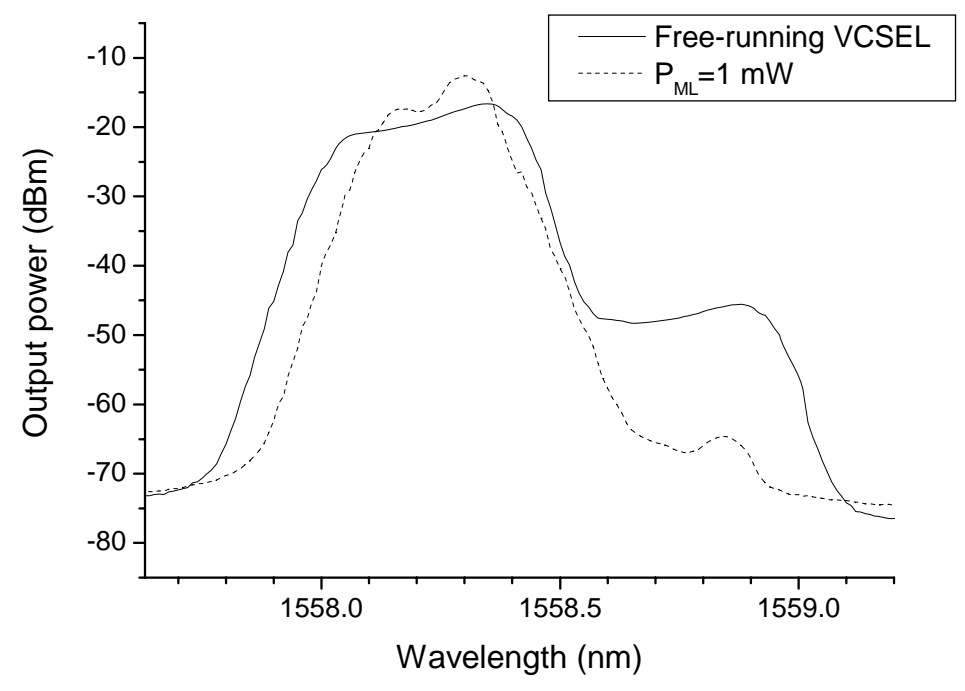

Figure 4. Optical spectrum of a gain-switched VCSEL for a repetition rate of $1 \mathrm{GHz}$ without (solid) and with (dashed) optical injection ( $\mathrm{P}_{\mathrm{ML}}=1 \mathrm{~mW}, \lambda_{\mathrm{ML}}=1558.26 \mathrm{~nm}$ and frequency detuning $\Delta v=5 \mathrm{GHz}$ ).

The optical spectrum becomes narrower because of the chirping reduction obtained by the injection locking of the VCSEL [1]. The mean spectrum width [12] is $14.8 \mathrm{GHz}$ for the free-running VCSEL and $9.3 \mathrm{GHz}$ for the VCSEL subject to optical injection with a frequency detuning $\Delta v=5 \mathrm{GHz}$. Therefore a reduction of $37 \%$ in the spectrum width is obtained by optical injection.

We now characterize the optical pulses obtained for different injection wavelengths and $\mathrm{P}_{\mathrm{ML}}=1 \mathrm{~mW}$. We show in Fig. 5 (a)-(c) the averaged shapes of pulses when $\lambda_{M L}=1558.58,1558.48$ and $1558.26 \mathrm{~nm}$, that correspond to a frequency detuning of $\Delta v=-35,-22.5$ and $5 \mathrm{GHz}$, respectively. Pulses obtained when $\lambda_{\mathrm{ML}}=1558.58 \mathrm{~nm}$ are very similar to the ones obtained without optical injection (full width at half maximum, FWHM=59.7 ps and maximum power, $\mathrm{P}_{\max }=0.79 \mathrm{~mW}$ ) because the frequency detuning, $\Delta v=-35 \mathrm{GHz}$, is very large. 

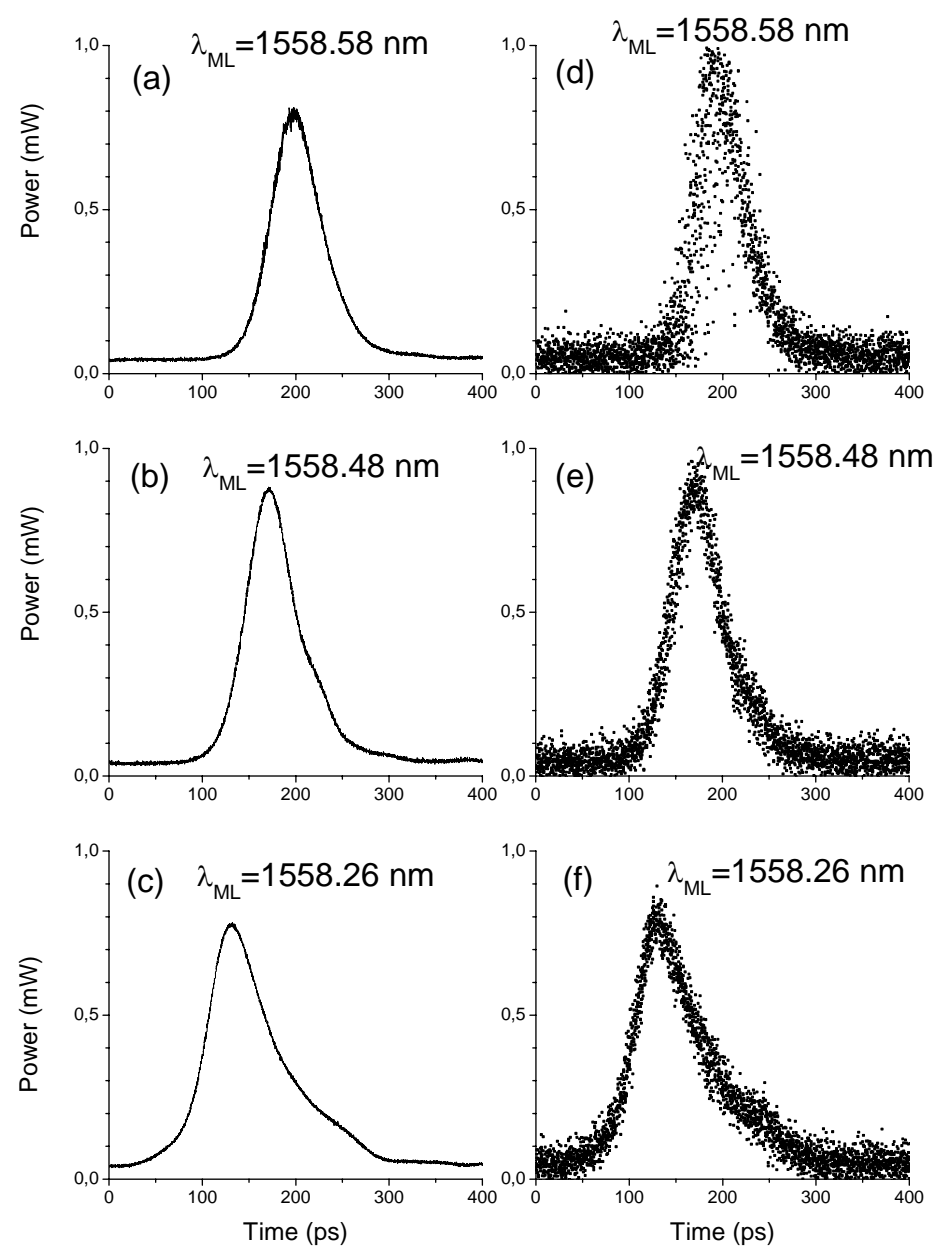

Figure 5. Averaged shapes of pulses generated by the VCSEL with optical injection for three values of $\lambda_{\mathrm{ML}}$ and $\mathrm{P}_{\mathrm{ML}}=1 \mathrm{~mW}$ are shown in parts (a), (b) and (c) that correspond to a frequency detuning of $\Delta v=-35,-22.5$ and $5 \mathrm{GHz}$, respectively. The corresponding oscilloscope traces are shown in parts (d)-(f).

In Fig. 6 we show the FWHM and $\mathrm{P}_{\max }$ as a function of $\lambda_{\mathrm{ML}}$ (upper horizontal axes) and of the corresponding frequency detuning, $\Delta v=v_{\mathrm{ML}}-v||$ (lower horizontal axis). Figs. 5 and 6 show that pulses become wider and more asymmetric as $\lambda_{\mathrm{ML}}$ approaches $\lambda_{\|}$. That detrimental fact is however compensated by the much smaller values of the timing jitter when $\lambda_{\mathrm{ML}}$ approaches $\lambda_{\|}$, as it is shown in Fig. 6 (a). That jitter is calculated as the standard deviation of the time at which the laser power crosses a level situated at one third of the maximum power. The decrease of the timing jitter is also illustrated in Figs. 5 (d)-(f) in which the oscilloscope traces of the pulses corresponding to Figs. 5 (a)-(c) are shown. Fig. 6 (a) shows that for a range from -20 to $27 \mathrm{GHz}$ optical injection reduces the $11.6 \mathrm{ps}$ jitter of the solitary VCSEL to a value below 4 ps. The averaged value of the jitter over that $47 \mathrm{GHz}$ detuning range is $3.45 \mathrm{ps}$ that results in a jitter reduction larger than $70 \%$. Over that $\Delta v$ range the averaged FWHM is 73 ps that produces just a slight increase in pulse width (22\%) with respect to the solitary case. It can also be seen that low values of the jitter also result in pulse broadening. A clear anticorrelation between $P_{\max }$ and pulse width is seen in Fig. 6 in the detuning range $-24 \mathrm{GHz}<\Delta v<30 \mathrm{GHz}$ with a value of the correlation coefficient of -0.93 . 

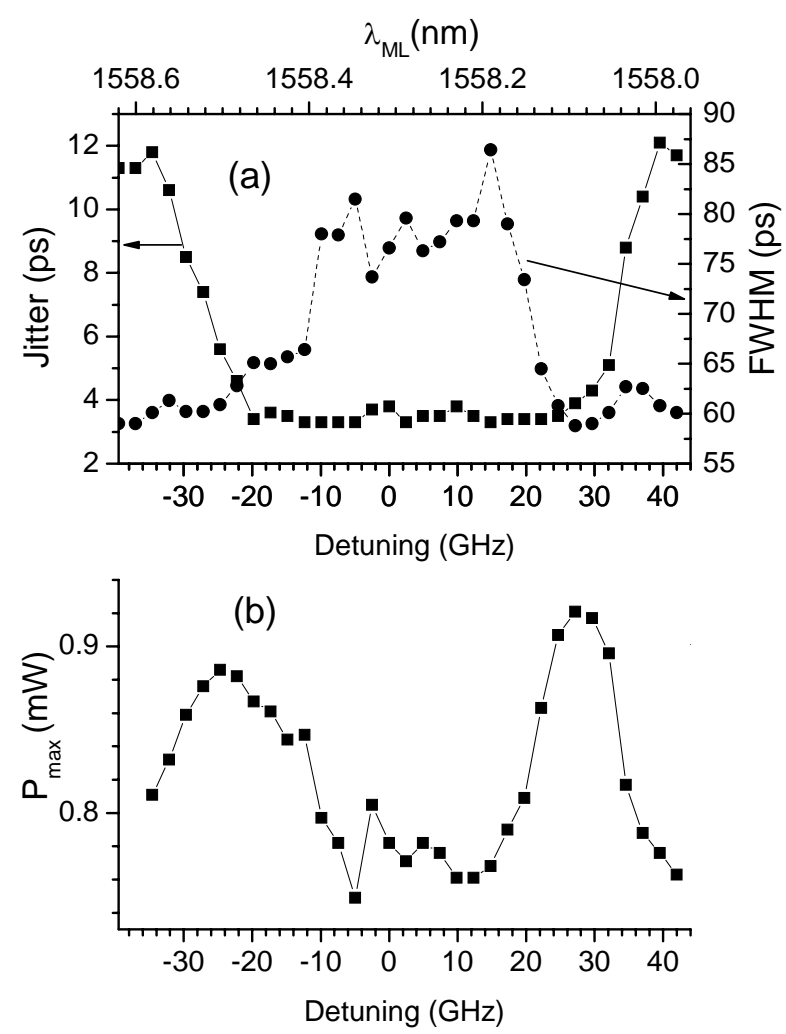

Figure 6. a) Jitter, FWHM and b) $\mathrm{P}_{\max }$ as a function of frequency detuning $\Delta v$ and $\lambda_{\mathrm{ML}}$ when the repetition frequency is $1 \mathrm{GHz}$ and $\mathrm{P}_{\mathrm{ML}}=1 \mathrm{~mW}$.

We have also considered the case of a lower repetition frequency. This case has a special interest in a simple OCDMA encoder based on optical delay lines [7,13], in which the length of the optical code, given by the ratio between the modulation period and the pulse width, is large. The number of possible users depends on that code length. Fig. 7 shows the results obtained for a $100 \mathrm{MHz}$ repetition frequency with $\mathrm{P}_{\mathrm{ML}}=1 \mathrm{~mW}$ and $\mathrm{I}_{\mathrm{BIAS}}=0.87 \mathrm{I}_{\mathrm{th}}$. The voltage in the PG is 2.5 $\mathrm{V}$ during $250 \mathrm{ps}$ and $0 \mathrm{~V}$ during the rest of the pulse. Again the optical injection decreases the timing jitter below its solitary value, $11 \mathrm{ps}$, over a wide $\Delta v$ range: when $-20 \mathrm{GHz}<\Delta v<22 \mathrm{GHz}$ the jitter is below 3 ps. The qualitative behavior of jitter, FWHM and $\mathrm{P}_{\max }$ is similar to the $1 \mathrm{GHz}$ repetition frequency case. Then optical injection can induce a low jitter optical pulse train that can be used in OCDMA systems with a code length of around 150 chips for a $100 \mathrm{MHz}$ repetition frequency.

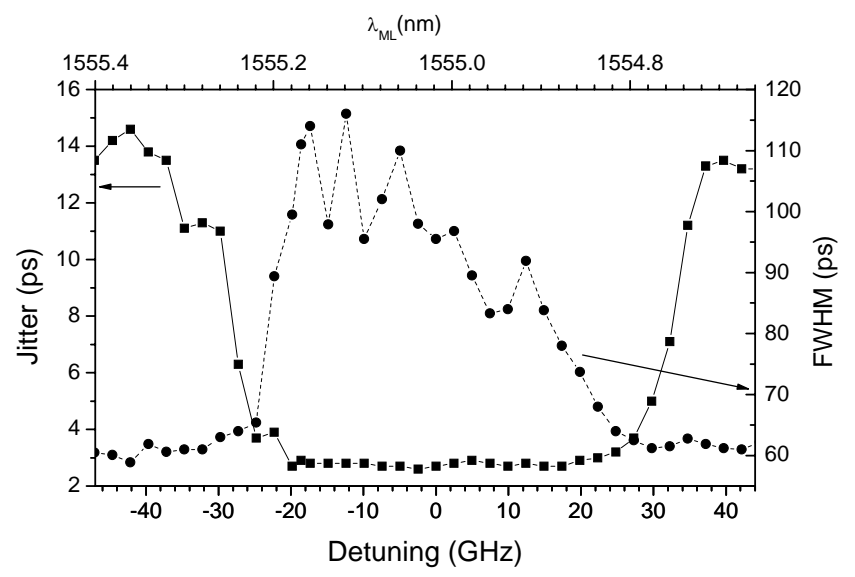

Figure 7. Jitter and pulse width as a function of $\Delta v$ when the repetition frequency is $100 \mathrm{MHz}$ and PML=1mW. 


\section{THEORETICAL RESULTS}

We perform a theoretical study by extending previous analysis [10] to take into account the two polarization modes. The pulse characteristics are obtained for the repetition rate of $1 \mathrm{GHz}$ considered in the experiments. The model utilized incorporates both spatial dependence of carrier density and optical field profiles [14]. A cylindrical weakly-index guided VCSEL with transverse modes $L P_{m n}$ is considered. The dynamical evolution of the complex field amplitude of each mode is given by

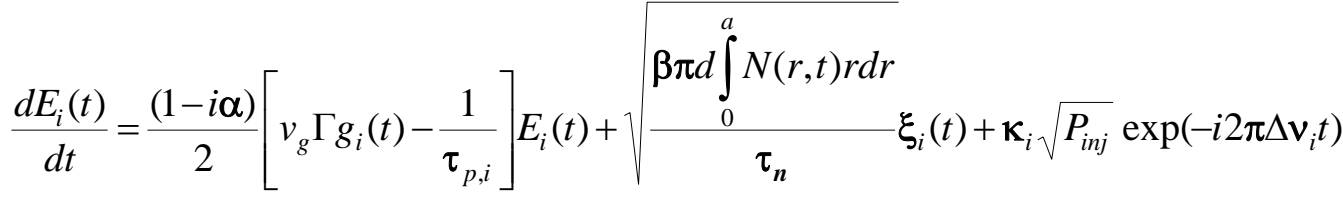

where $g_{i}(t)$ and $\tau_{p, i}$ are the modal gain and losses, respectively, of that mode, $v_{g}$ is the group velocity, $\Gamma$ is the longitudinal confinement factor, $a$ is the radius of the waveguide, $d$ is the active region thickness, $\tau_{\mathrm{n}}$ is the carrier lifetime, $\Gamma$ is the longitudinal confinement factor, $\alpha$ is the line-width enhancement factor, $\beta$ is the spontaneous emission factor, $\xi$ is a complex white Gaussian noise term of zero mean, and $N(r, t)$ is the carrier density. The last term of Eq. (1) accounts for optical injection. $P_{i n j}$ is the injected power, $\kappa_{i}$ is the injection parameter into the $i$-mode and $\Delta v_{i}=v_{i n j}-v_{i}$ is the frequency detuning of the injected signal from the frequency of the $i$-mode. Results in this work are given in terms of the detuning $\Delta v=v_{i n j}-v$ with respect to the frequency of the lowest threshold mode $v$.

The modal gain of the $i$-mode is given by $g_{i}(t)=\int_{0}^{\infty} \psi_{i}^{2}(r) A\left(N(r, t)-N_{t}\right) r d r /<\psi_{i}^{2}>$, where the symbol $<>$ is used for denoting the following integral, $\left\langle f>=\int_{0}^{\infty} f(r) r d r, r\right.$ is the modulus of the spatial vector in the active layer, $\psi_{i}(r)$ is the electrical field profile of the $i$-mode, $N_{t}$ is the carrier density at transparency, and $A$ is the gain coefficient. The modal gain represents the degree of spatial overlap between the mode intensity profile and the carrier distribution. The evolution of the carrier density profile, $N(r, t)$, is given by the carrier continuity equation applied to the active region:

$$
\frac{\partial N(r, t)}{\partial t}=\frac{D}{r} \frac{\partial}{\partial r}\left(r \frac{\partial N(r, t)}{\partial r}\right)-\frac{N(r, t)}{\tau_{n}}+\frac{j(r, t)}{e d}-\sum_{i} a_{i} \psi_{i}^{2}(r) g_{i}(t) P_{i}(t)
$$

where $D$ is the diffusion coefficient, $j(r, t)$ is the current density injected in the active region, $e$ is the electron charge, and $a_{i}=v_{g} \Gamma /\left(2 \pi d<\psi_{i}^{2}>\right)$. We consider a uniform injection of current over a disk of radius $s$ and then, $j(r)=j$ if $r<a$, and $j(r)=0$ elsewhere. The evolution of the electrical field is found by integrating Eqs. (1) and (2). A complete description of the model together with the values of the parameters can be found elsewhere $[10,14]$.

We consider that the VCSEL emits only in the two linear polarizations of the fundamental mode $L P_{01}$ at a wavelength $1558.3 \mathrm{~nm}$ for the dominant parallel polarization and $1558.79 \mathrm{~nm}$ for the orthogonal polarization. We take into account this in the modeling by considering that the modal losses of these two polarizations are much smaller than those of the rest of the modes. The modal losses of the parallel and orthogonal polarizations are taken as $\tau_{p, 1}=1.5$ ps and $\tau_{p, 2}=1.48$ ps, respectively. We also consider the same field profile for the two polarizations, $\psi_{1}(r)=\psi_{2}(r)$, corresponding to the fundamental transverse mode. Under continuous wave operation the orthogonal polarization is suppressed more than 30 $\mathrm{dB}$ with respect to the parallel polarization in agreement with the experimental results.

A square-wave modulation is applied to the VCSEL with a repetition rate of $1 \mathrm{GHz}$ and duty cycle of $50 \%$. The laser is switched from below threshold to above threshold condition. We consider a bias current $j_{b}=0.5 j_{\text {th }}$ and an on-state current $j_{\text {on }}=2 j_{\text {th }}$ in order to have short pulses with a good extinction ratio. The power of the injected signal $P_{i n j}$ is given in $d B s$ 
with respect to the power of the solitary laser $P_{\text {on }}$ that corresponds to the on-state current $j_{\text {on }}$. The light is injected parallel to the dominant linear polarization. The optical spectrum of the free-running VCSEL calculated with a resolution of 1 $\mathrm{GHz}$ is shown in Fig. 8. Spectral broadening due to chirping effects is obtained. The main peak corresponds to the parallel polarization. The peak that appears at longer wavelengths corresponds to the orthogonal polarization that is suppressed only $7.5 \mathrm{~dB}$ with respect to the parallel polarization (solid line of Fig. 8). Then the side mode suppression ratio is reduced more than $20 \mathrm{~dB}$ due to the gain-switching operation of the VCSEL in agreement with the experimental results shown in Fig. 3. The optical spectrum of the VCSEL subject to optical injection is also shown in Fig. 8 (dashed line). The optical injection is such that the power of the tunable laser is $P_{i n j}=-34 \mathrm{~dB}$ and the frequency detuning of the injected signal with respect to the parallel polarization is $\Delta v=5 \mathrm{GHz}$. Fig. 8 shows that optical injection produces a narrowing of the optical spectrum and an improvement of the side mode suppression ratio of $13 \mathrm{~dB}$. The optical spectrum becomes narrower because of the chirping reduction obtained by the injection locking of the VCSEL. A reduction of $29 \%$ in the mean spectrum width of the parallel polarization is obtained by optical injection with a frequency detuning $\Delta v=5 \mathrm{GHz}$. All these results are in qualitative agreement with the experimental results shown in Fig. 4.

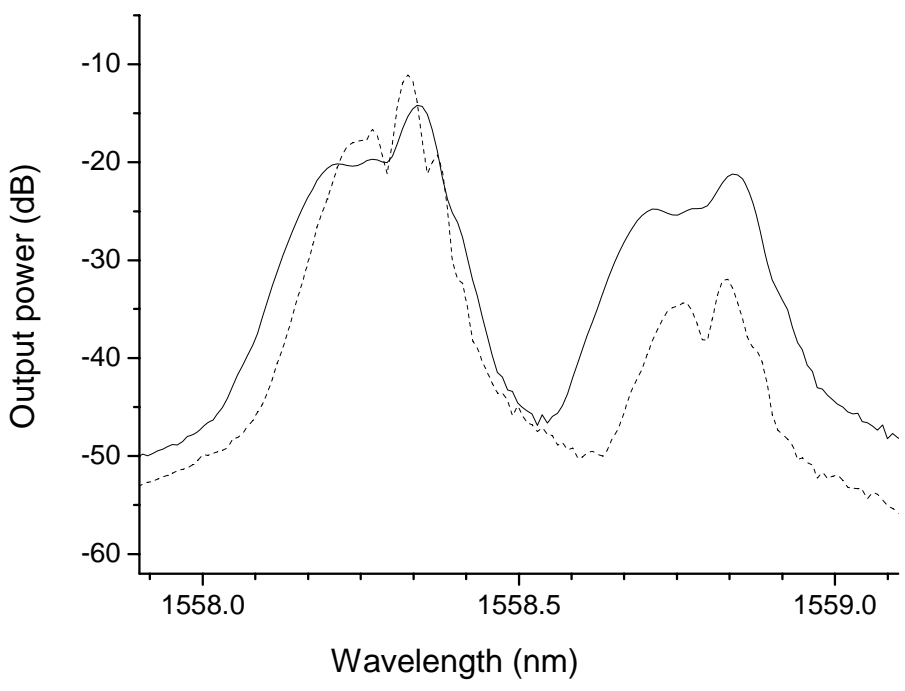

Figure 8. Theoretical results for the optical spectrum of a gain-switched VCSEL at a repetition rate of $1 \mathrm{GHz}$ without (solid) and with (dashed) optical injection ( $P_{i n j}=-34 \mathrm{~dB}$ and frequency detuning $\left.\Delta v=5 \mathrm{GHz}\right)$.

We now analyze the effect of optical injection on timing jitter, pulse width (full width at half maximum, FWHM), and peak power, $P_{\max }$. We only consider the parallel polarization component of the pulses in order to compare with the experimental results. The jitter is obtained by using a reference value situated at $P_{\max } / 3$. In Fig. 9 we show the jitter and FWHM as a function of the frequency detuning $\Delta v$ for an injection power of $-34 \mathrm{~dB}$. A good qualitative agreement is obtained between the theoretical and the experimental results shown in Fig. 6a. It is found that for a detuning range from -10 to $10 \mathrm{GHz}$ optical injection reduces the 13.6 ps jitter of the solitary VCSEL to a value below 7 ps. The averaged value of the jitter over that $20 \mathrm{GHz}$ detuning range is 5.2 ps that results in a jitter reduction larger than $60 \%$. When the injected power $P_{i n j}$ is increased, jitter reduction can be achieved for a greater detuning range. However, the oscillations of the jitter (see Fig. 9 for small negative values of $\Delta v$ ) also increase. Over the detuning range from -10 to $10 \mathrm{GHz}$ the averaged FWHM is 76 ps that produces a slight increase of $15 \%$ in pulse width with respect to the solitary case. It can also be seen that low values of the jitter result in pulse broadening. 


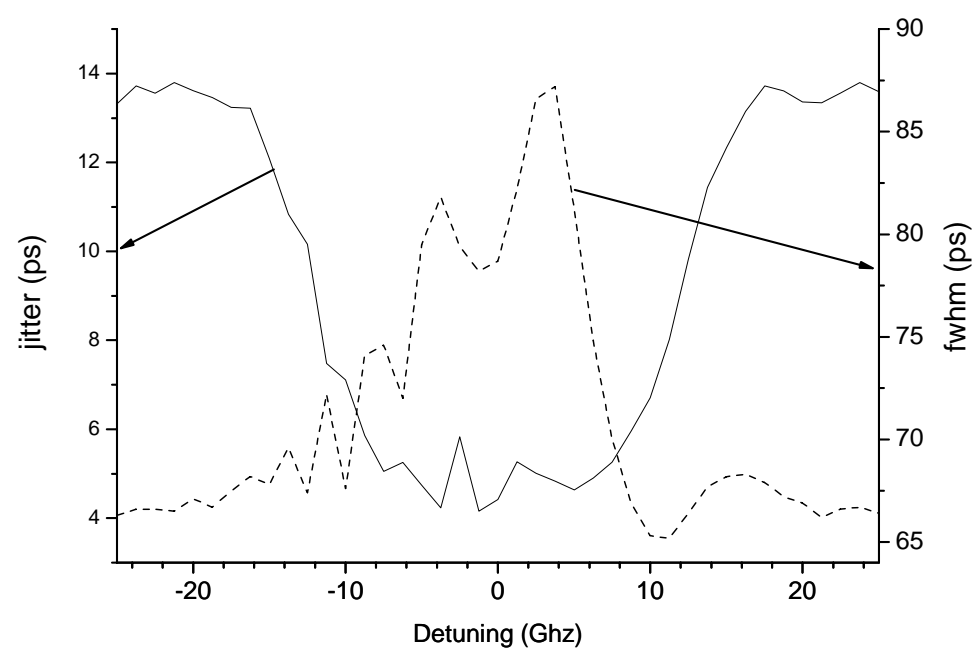

Figure 9. Theoretical results for jitter and pulse width as a function of frequency detuning $\Delta v$ when the repetition frequency is $1 \mathrm{GHz}$ and $\mathrm{P}_{\mathrm{inj}}=-34 \mathrm{~dB}$.

We show in Fig. 10 the peak power as a function of the frequency detuning $\Delta v$ for an injection power of $-34 \mathrm{~dB}$. The theoretical result is in good agreement with the experimental result shown in Fig. 6b. A clear anticorrelation between $\mathrm{P}_{\max }$ and pulse width is seen in Figs. 9 and 10 in the detuning range $-15 \mathrm{GHz}<\Delta v<15 \mathrm{GHz}$ with a value of the correlation coefficient of -0.93 . This value is very close to the one obtained from the experimental results.

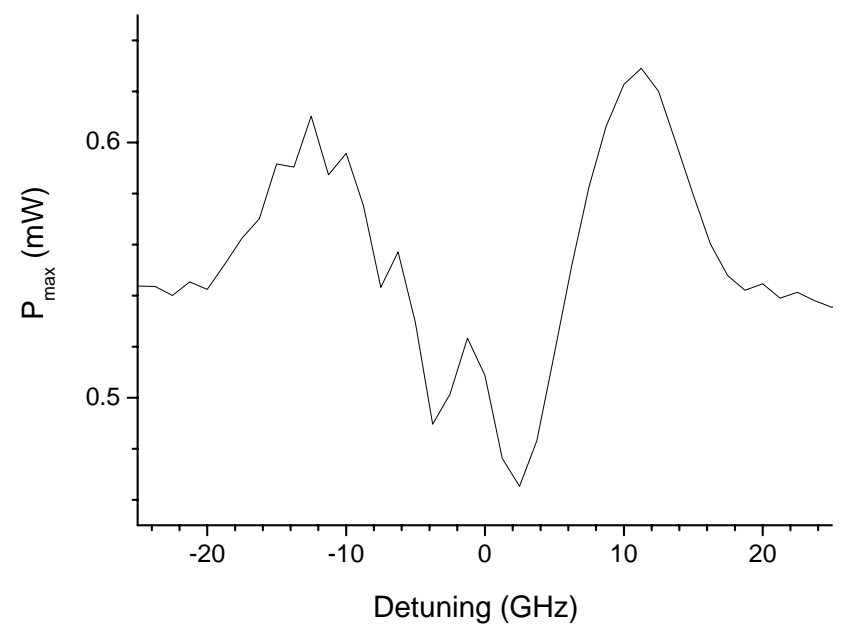

Figure 10. Theoretical results for peak power $\mathrm{P}_{\max }$ as a function of frequency detuning $\Delta v$ when the repetition frequency is 1

$\mathrm{GHz}$ and $\mathrm{P}_{\text {inj }}=-34 \mathrm{~dB}$.

\section{CONCLUSIONS}

We have investigated the effect of optical injection on the characteristics of optical pulses generated by gain-switching a $1550 \mathrm{~nm}$ single transverse mode VCSEL. Under continuous wave operation the VCSEL emits in a linear polarization along the whole current range. We have performed an experimental analysis of the effect of external optical injection on the timing jitter, maximum power, and pulse width of optical pulses for two repetition rates, $100 \mathrm{MHz}$ and $1 \mathrm{GHz}$, and for different values of the detuning between the frequency of the optical injection and the VCSEL. The qualitative behavior is similar for both repetition rates. In the $1 \mathrm{GHz}$ case we have obtained jitter reductions larger than $70 \%$ over a 
$47 \mathrm{GHz}$ detuning range centered around the VCSEL lasing frequency. That jitter reduction is accompanied by a slight increase in pulse width with respect to the free-running case. A clear anticorrelation between peak power and pulse width is obtained. We have also performed a theoretical study by using a model that incorporates both spatial dependence of carrier density and optical field profiles. The two polarization modes of the fundamental transverse mode are taken into account in the model. We have obtained timing jitter, maximum power, and pulse width of optical pulses for a repetition rate of $1 \mathrm{GHz}$, and for different values of the detuning between the frequency of the optical injection and the VCSEL. The results obtained from numerical simulations for pulse characteristics are in good qualitative agreement with the experimental results.

\section{ACKNOWLEDGEMENTS}

The authors acknowledge support from the Ministerio de Ciencia e Innovación under projects TEC2009-14581-C02-01 and TEC2009-14581-C02-02.

\section{REFERENCES}

[1] Chang, C. H. Chrostowski, L. and Chang-Hasnain, C. J., “Injection locking of VCSELs,” IEEE J. Selec. Top. In Quantum Electron. 9(5), 1386-1393 (2003).

[2] Lau, E. K. Zhao, X. Sung, H. K. Parekh, D. Chang-Hasnain, C. and Wu, M. C., "Strong optical injection-locked semiconductor lasers demonstrating $>100-\mathrm{GHz}$ resonance frequencies and 80-GHz intrinsic bandwidths," Opt. Express 16(9), 6609-6618 (2008).

[3] Koyama, F., “Recent advances of VCSEL Photonics,” J. Lightwave Technol. 24, 4502-4513 (2006).

[4] Ortsiefer, M. Baydar, S. Windhorn, K. Bohm, G. Rosskopf, J. Shau, R. Ronneberg, E. Hofmann, W. and Amann, M. C., “2.5-mW single-mode operation of $1.55-\mu \mathrm{m}$ buried tunnel junction VCSELs,” IEEE Photon. Technol. Lett. 17(8), 1596-1598 (2005).

[5] Kim, J. J. Kim, K. H. Lee, M. H. Lee, H. S. Lee, E. H. Kwon, O-K. Roh, J.and Yo, B-S., “2.5 Gb/s hybrid single-mode and multimode fiber transmission of $1.5 \mu \mathrm{m}$ wavelength VCSEL,” IEEE Photon. Technol. Lett. 19(5), 297-299 (2007).

[6] Wang, X. Wada, N. Hamanaka, T. Miyazaki, T. Cincotti, G. and Kitayama, K. “OCDMA over WDM Transmission,” Transparent Optical Networks, 2007. ICTON '07. 9th International Conference on, vol. 1, 110113 (2007).

[7] Consoli, A. Esquivias, I. López-Hernández, F.J. Balle, S. and Mulet, J., "Pulse Generation from Gain-Switched Vertical Cavity Surface Emitting Laser at $1.5 \mu \mathrm{m}$,” Advances in Electronics and Micro-Electronics, 2008. ENICS '08. International Conference on, $72-77$ (2008).

[8] Seo, D. S. Kim, D. Y. and Liu, H. F., "Timing jitter reduction of a gain-switched DFB laser by external injection-seeding,” Electron Lett. 32, 44-45 (1996).

[9] Guignard, C. Anandarajah, P. M. Clarke, A. Barry, L. P. Vaudel, O. and Besnard, P., "Experimental investigation of the impact of optical injection on vital parameters of a gain-switched pulse source,” Opt. Comm. 277, 150-155 (2007).

[10] Noriega, J. M. Valle, A. and Pesquera, L., “Timing jitter reduction in gain-switched VCSELs induced by external optical injection,” Opt. Quant. Electron. 40, 119-129 (2008).

[11] Balle, S. De Pasquale, F. Abraham, N. B. and San Miguel, M., "Statisticts of the transient frequency modulation in the switch-on of a single-mode semiconductor laser,” Phys. Rev. A 45, 1955-1966 (1992).

[12] Dellunde, J. Torrent, M. C. Sancho, J. M. and San Miguel, M., "Frequency Dynamics of Gain-Switched Injection-Locked Semiconductor Lasers,” IEEE J. Quantum Electron. 33, 1537-1542 (1997).

[13] Prucnal, P. R. Santoro, M. A. and Fan, T., "Spread spectrum fiber-optic local area network using optical processing,” J. Lightwave Technol. 4, 547-554 (1986).

[14]Valle, A., "Selection and modulation of high-order transverse modes in VCSELs,” IEEE J. Quantum Electron. 34, 1924-1932 (1998). 